\title{
LA CIUDADELA (1938) DE KING VIDOR: LUCES Y SOMBRAS EN EL EJERCICIO DE LA PROFESIONALIDAD
}

\section{The Citadel (1938) by King Vidor: Lights and shadows in the practice of professionalism}

\author{
Palmer José HERNÁNDEZ-YÉPEZ \\ Facultad de Medicina Humana. Universidad Privada Norbert Wiener (Perú). \\ Autor para correspondencia: Palmer José Hernández-Yépez. \\ Correo electrónico: palmerxvii@gmail.com
}

Recibido el 23 de marzo de 2020

Aceptado el 14 de abril de 2020

\section{Resumen}

La ciudadela (1938) de King Vidor, es una película que narra la vida profesional del doctor Andrew Manson, desde su entusiasmado inicio como médico recién graduado en un pueblo minero en Gales, donde labora apasionadamente en diferentes casos sin importar las circunstancias, hasta su llegada a Londres donde ve distorsionado su propósito al adentrarse en la élite de la ciudad. Con la ayuda de su esposa Christine, tratará de recobrar el significado de la medicina. A través del filme se exhibe la ligera línea que divide el buen ejercicio de la profesionalidad. Por ello, en el presente trabajo se reflexiona sobre la medicina como profesión, su relación con la sociedad y se destaca la importancia de la educación en humanidades para el fortalecimiento del discernimiento ético en los futuros profesionales en salud.

Palabras clave: profesionalismo; ética médica; relación médico-paciente.

\section{Abstract}

The Citadel (1938) by King Vidor, is a film that narrates the professional life of Dr. Andrew Manson, from his enthusiastic start as a recently graduated doctor in a mining town in Wales, where he works passionately in different cases regardless of the circumstances, until his arrival in London where he sees his purpose distorted as he enters the elite of the city. With the help of his wife Christine, he will try to regain the meaning of medicine. Throughout the film the thin line that 
divides the good exercise of professionalism is exhibited. For this reason, this paper reflects on medicine as a profession, its relationship with society, and highlights the importance of education in the humanities for strengthening ethical discernment in future health professionals.

Keywords: professionalism; medical ethics; doctor-patient relationship.

\section{Ficha técnica}

Título original: The Citadel.

Otros títulos: La Ciudadela (España).

País: Estados Unidos.

Año: 1938.

Director: King Vidor.

Música: Louis Levy.

Fotografía: Harry Stradling Sr.

Montaje: Charles Frend.

Guion: Ian Dalrymple, Frank Wead, Elizabeth Hill. Basado en la novela de A.J. Cronin.

Intérpretes: Robert Donat, Rosalind Russell, Ralph Richardson, Rex Harrison, Emlyn Williams, Penelope Dudley-Ward, Francis L. Sullivan, Mary Clare, Cecil Parker, Nora Swinburne, Edward Chapman,...

Color: blanco y negro.

Duración: 110 minutos.

Género: drama, medicina.

Idioma original: inglés.

Productoras: Metro-Goldwyn-Mayer (MGM) Sinopsis: «Andrew Manson, un médico joven y entusiasta, toma su primer trabajo en una ciudad minera galesa y comienza a preguntarse por la tos persistente que tienen muchos de los mineros. Cuando sus intentos de demostrar su causa se ven frustrados, se muda a Londres. Su nueva práctica le va mal. Pero cuando un amigo le muestra cómo hacer una práctica lucrativa a partir de hipocondríacos ricos, le sorprenderá cuál es realmente la verdad de ser médico» (FilmAffinity).

\section{Enlaces:}

https://www.imdb.com/title/tt0029995

https://www.filmaffinity.com/us/film899073.html

\section{Tráiler en inglés}

\section{Introducción}

El sentido de la medicina tiene su base en la vocación, y su integridad en el servicio. En la práctica diaria, los profesionales en salud, particularmente los médicos, deben realizar juicios clínicos de valor acerca de cuál de las opciones disponibles es la mejor para un paciente en particular. Para ello, se basan en los cuatro principios de la bioética: autonomía, beneficencia, no maleficencia y justicia ${ }^{1}$. Esto conducirá a que los médicos proporcionen razones coherentes en juicios de valor con los cuales informan sus decisiones clínicas.

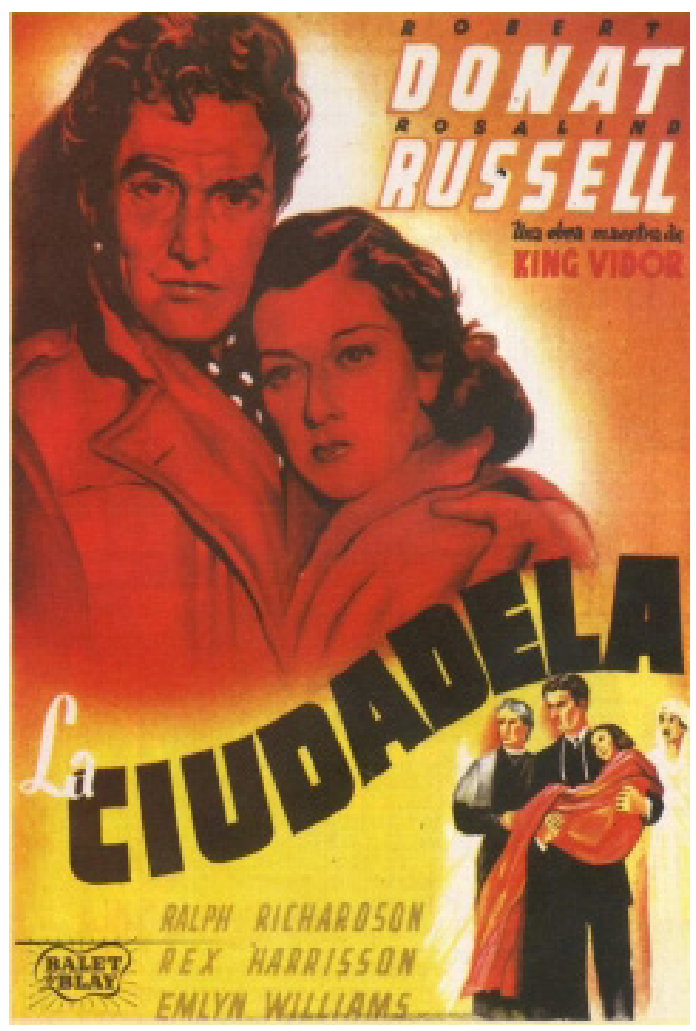

Cartel español.

El filme La ciudadela (1938) de King Vidor trata acerca del ejercicio profesional del doctor 
Andrew Manson (Robert Donat), quien recién graduado cómo médico es asignado en el pueblo minero ficticio de Blaenely en Gales. Allí tiene la labor de tratar a los mineros que en su mayoría contraen neumonías producto del polvo de carbón y de sílice. Sin embargo, a pesar de sus objetivos científicos nobles, encuentra resistencia en su investigación, por parte de colegas y los propios mineros.

Posteriormente se muda a Londres donde su propósito se erosiona al introducirse en una clínica donde solo se atiende a la élite londinense, siendo el objetivo de esta entidad carente de ética sacar todo el dinero posible de los bolsillos de los pacientes. Con la ayuda de su esposa Christine (Rosalind Russell) intentará volver por el camino original de la noble profesión que es la medicina.

En el presente artículo, se propone reflexionar sobre la medicina como profesión, su relación con la sociedad a través de la salud pública y la salud ocupacional. Asimismo, se resaltan algunos elementos negativos que están presentes en la práctica médica y se proponen soluciones a través de la educación para que no sigan ocurriendo.

\section{La medicina como profesión}

Para entender la medicina como profesión, primero se debe definir a la profesión por sí sola, lo cual no es tarea fácil. Etimológicamente deriva del latín professio, que refiere a la acción y efecto de profesar, ya sea una creencia religiosa, un sentimiento, o una carrera; es en esta última que nos vamos a centrar. La profesión está unida al componente vocación, pues implica un servicio a los demás y tiene como base de interés vital a la persona. También posee una base de conocimiento científico distintiva, la cual se mantiene constantemente actualizada y determina sus propios estándares. Por último, tiene una relación especial con aquellos a quienes sirve: clientes, pacientes, etc. lo cual indispensablemente la hace tener principios particulares cuya base es la ética, siendo responsable ante sus usuarios y ante la profesión misma ${ }^{2,3}$.

Por lo general, los médicos atienden pacientes, diagnostican, prescriben y establecen tratamientos. Ese aspecto de atención clínica supone un rol importante, pues constituye los cimientos de la relación médico-paciente al entablar un lazo de compromiso y compasión por el paciente, y se solidifica con la confianza de este último, siempre y cuando aquel profesionalismo sea correctamente ejercido. Sin embargo, la labor de los médicos no se restringe al ámbito clínico, o idealmente no debería ser así, pues el médico también realiza investigaciones y se dedica a la docencia de futuros profesionales en salud. Asimismo, los médicos deben tener competencias y habilidades en la gestión de recursos, pues algunos no solo atienden pacientes sino, en su ámbito más general, familias o hasta comunidades enteras. Entonces, el manejo de recursos no solo se limita en términos de financiamiento, sino también en el uso apropiado del tiempo, habilidades e instalaciones disponibles. Los recursos siempre han sido y seguirán siendo finitos ${ }^{4}$. Frente a esto, el médico constantemente se encuentra tratando de resolver el clásico dilema de cómo hacer lo mejor para un paciente sin perjudicar a otro.

Los rasgos representativos mencionados anteriormente se reflejan al inicio del filme en el protagonista, el Dr. Manson, quien recién graduado de la carrera, se encuentra entusiasmado por empezar a atender diferentes casos, poco le importa el momento del día o la distancia de los casos que debe atender. A medianoche acude presto al llamado de un caso de una mujer mayor con sospecha de fiebre tifoidea (Foto 1) o sino cuando acude a atender partos en lugares distantes del pueblo. 


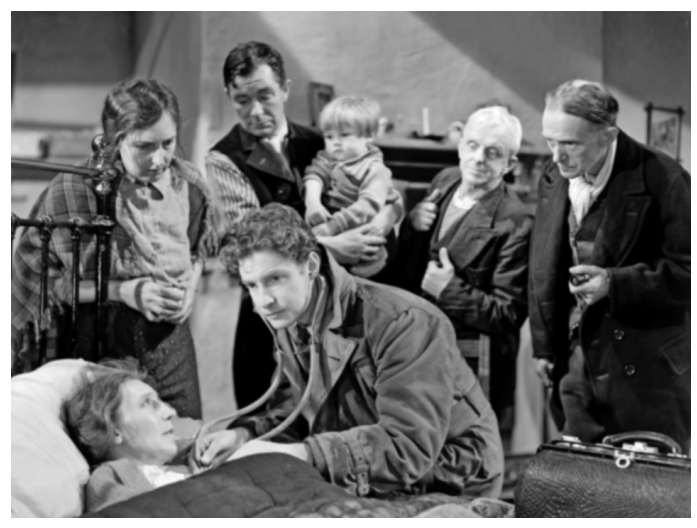

Foto 1. El Dr. Manson atiende con empeño a su primera paciente.

Otro claro ejemplo de aquel ímpetu que debe caracterizar a los médicos se da cuando, al no contar con un ambiente adecuado para poder analizar más a fondo las muestras de sus pacientes, implementa un laboratorio en su casa junto a su esposa con el propósito de investigar muestras de esputo de los mineros con neumonía (probablemente tuberculosis, según él refiere), además de muestras de polvo de carbón y sílice (Foto 2).

\section{Medicina y sociedad}

Es imposible afirmar que la medicina que se practicaba hace un siglo es la misma que se practica en la actualidad, pues el precipitado desarrollo de la tecnología biomédica, con descubrimientos e inventos cada vez más admirables, ha hecho que la medicina haya cambiado continuamente a lo largo de la historia ${ }^{5}$. Esto ha conllevado a reflexionar sobre la práctica médica. Es un hecho que la medicina no puede existir individualmente y por eso debe estar en relación con la sociedad, pues a esta última es a quien sirve ${ }^{6}$. Entonces, frente a este panorama, ¿Es necesario que la medicina deba redefinirse continuamente en función de las necesidades sociales?

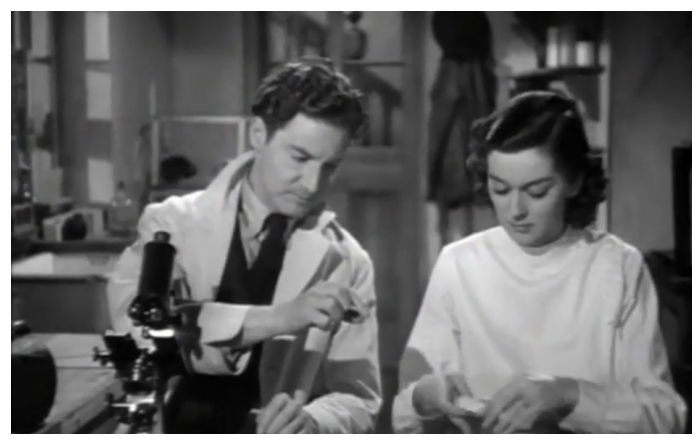

Foto 2. El Dr. Manson con su esposa Christine analizando muestras de esputo de los mineros.

La medicina cumple un rol importante no solo en el individuo, sino en la sociedad. Y sin esta última, la medicina no puede avanzar; pues los médicos son agentes de cambio social. Ya desde el siglo XIX, en Alemania se había puesto énfasis en este aspecto con las ideas de Virchow, quien afirmaba que los médicos son los abogados de los pobres y los problemas sociales no son ajenos a ellos; o a mediados de los sesenta en Inglaterra con McKeown y Lowe, quienes sostenían que la medicina social busca el estudio de las necesidades médicas en la sociedad y esta a su vez está en estrecha correlación con la epidemiología, ${ }^{7,8}$. Al mencionar lo presente, no se debe excluir tampoco a la Medicina Basada en la Evidencia (MBE) la cual consiste en el empleo de la mejor evidencia disponible y conlleva el empleo analizado y comparado de la literatura científica9.

En el filme estos conceptos se plasman cuando el Dr. Manson atiende a un niño con sarampión y la madre le afirma que había asistido al colegio durante esos días; por lo tanto, el doctor realiza un seguimiento hasta la escuela para comprobar si existían posibles casos adicionales (Foto 3 ). Asimismo, la MBE puede apreciarse cuando un minero sufre una quemadura en la pierna y el $\mathrm{Dr}$. Manson afirma que se debe aplicar un antiséptico (pues así lo sugieren los últimos estudios de la época) para evitar alguna posible infección; en 
contraste, la enfermera del pueblo quien siempre ha atendido estos casos afirma que basta nomás con aplicar aceite de romero y dejar reposar, increpándole por los estudios mencionados que nadie entiende por estar en latín.

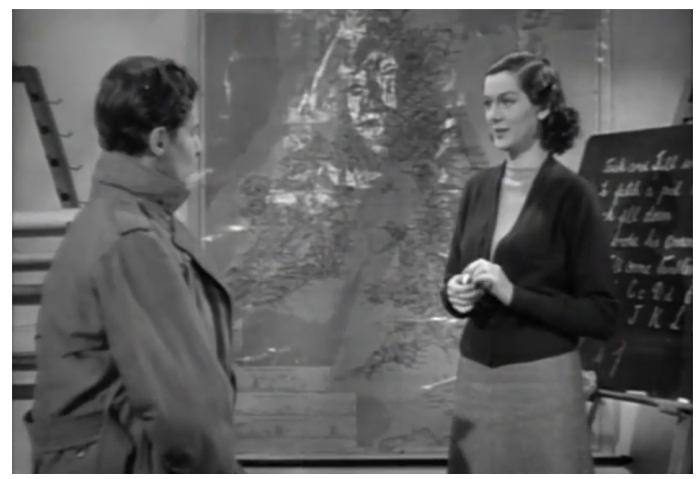

Foto 3. El Dr. Manson platicando con la profesora para verificar que no existan más casos de sarampión.

Lo expuesto anteriormente, tanto la epidemiologia y el triunfo de la evidencia sobre la experiencia (sin menospreciar a esta última) nos lleva a hablar del siguiente punto.

Salud pública y salud ocupacional, una estrecha relación

La salud pública en términos generales se refiere a las acciones colectivas dirigidas a mejorar la salud de la población; y para ello promueve esfuerzos comunitarios con el propósito del control de infecciones en la comunidad, saneamiento del medio ambiente, y la organización de los servicios de prestación de salud para un diagnóstico precoz. Es decir, promueve una cultura de prevención al fomentar la educación en las personas sobre los principios de higiene personal y así garantizar un conocimiento básico para desarrollar buenas prácticas en salud. Para lograr este propósito, la epidemiología no es únicamente un instrumento sino la base de la salud pública ${ }^{10}$.

Por otro lado, la medicina ocupacional se ocupa de analizar las condiciones laborales y su impacto en la salud de los trabajadores. Asimismo, enfatiza la prevención, el diagnóstico y tratamiento de las enfermedades causadas o exacerbadas por las condiciones de trabajo; así como las repercusiones médico-legales de esta. Así, la medicina ocupacional aborda de manera integral la relación entre el trabajo y la salud, al promover la capacidad y desempeño laboral de los trabajadores y velar por la salud de ellos ${ }^{11,12}$

Entonces, con lo ya expuesto, existe una relación estrecha entre la salud ocupacional y la salud pública. Pues si bien la primera se limita al ámbito laboral la otra vela por la sociedad en general, y el trabajo es un componente fundamental en la sociedad. No se puede hablar de medicina, sin hablar de sociedad y no se puede hablar de sociedad sin hablar de trabajo.

No se puede afirmar en su totalidad que el protagonista sea un médico ocupacional, pues su labor no se limita solo a la atención de los trabajadores en su jornada laboral, sino que se extiende al pueblo minero donde está asignado. Desde esta perspectiva cumple más el rol de un médico familiar o incluso el de un médico salubrista. En el filme se puede apreciar que el Dr. Manson escribe constante correspondencia hacia el Departamento de Sanidad solicitando que cambien el pozo principal del pueblo pues es evidente que está contaminado y por ello la gente se enferma, pero al no recibir respuesta al mismo tiempo que más personas contraían enfermedades, decide, en un acto altruista y a la vez vehemente, destruir el pozo y así obligatoriamente se deba construir uno nuevo.

\section{Arrogancia y medicina: una mala combinación}

Mientras el Dr. Manson trata de ayudar a sus pacientes de la mina de forma poca ortodoxa se ve frente a la negativa de no solo diferentes colegas sino hasta de los mismos pacientes. Los trabajadores únicamente desean que les traten los síntomas mas no la enfermedad pues ellos necesitan de su jornada para vivir. Un médico colega (ya avanzado de edad) le dice: «No intente derribar 
los muros de Babilonia en un día», en referencia a los métodos innovadores propuestos.

Mientras más conocimiento, más dudas, y a veces más arrogancia. No debe confundirse la arrogancia con el modelo paternalista de la relación médico-paciente, el cual ha estado presente por muchos siglos e incluso sigue siendo practicado por algunos doctores en la actualidad. Independientemente a ello, el respeto y prestigio otorgado a los médicos puede fomentar la arrogancia en algunos ${ }^{13}$. En la actualidad, el sistema de atención médica ha despersonalizado la relación médico-paciente y ha intensificado ya no una arrogancia personal del médico sino una arrogancia del sistema, y esto es producto de que el paciente no es visto como una persona sino como una enfermedad a tratar de la cual se puede obtener una rentabilidad.

No se debe afirmar que las conclusiones alcanzadas en ausencia de hechos confiables sean por sí mismas arrogantes, mas aun en medicina, en donde nada es absoluto, pues es una conclusión (diagnóstico) que se da frente a un paciente, una persona. Y como diría Osler: «El buen médico trata la enfermedad; el gran médico trata al paciente que tiene la enfermedad." La arrogancia se presenta cuando quienes toman varias decisiones, en ausencia de datos adecuados, no reconocen ni admiten aquella incertidumbre de su información. Los buenos médicos siempre están concientes de qué tan poco ellos saben ${ }^{14}$. Si así es el paradigma de la arrogancia de la experiencia, imaginémonos cómo es la letalidad de la arrogancia de la ignorancia, la cual casi nunca es mencionada.

\section{Dinero y medicina}

Con el auge del sector privado en el sistema de salud en las últimas décadas se podría intuir que el servicio de prestación de salud se está tornando en un mercado en donde las inversiones y las ganancias económicas priman sobre la atención que se le ofrece al paciente. Aquel que estudia medicina simplemente para enriquecerse, tarde o temprano descubrirá que alguna otra ocupación puede satisfacer aquella necesidad de mejor manera, aunque eso no significa que los médicos tengan que vivir una vida de penurias y pobreza.

Aun así aceptando que la prestación de salud pueda ser una forma de negocio, esto abre las puertas a otra materia: el conflicto de intereses, de los cuales los médicos no están exentos de caer, ya sea a través de farmacéuticas y laboratorios privados, investigación clínica, o sino por el ahorro de recursos propios de la entidad de salud. Todo esto puede alterar las decisiones clínicas que propone el médico ${ }^{15,16}$.

Independientemente de cómo puede ser la tendencia del sistema de salud en los países, no se puede permitir que el mercado determine quién recibirá atención y tratamiento médico, pues la salud (como derecho fundamental) está por encima de la economía; y por lo mismo el mercado no debe definir la ética de la profesión médica.

En el filme esto se plasma cuando el Dr. Manson migra a Londres donde apertura su consultorio persistiendo, inicialmente, su espíritu caritativo. Conforme avanza el tiempo, mediante un colega de la facultad, se introduce como médico en la élite londinense, quienes en su mayoría son personas con buena salud, pero hipocondriacos. Trabaja en una clínica poco ética donde ejercen médicos que afirman ser especialistas en distintas áreas, cuando en realidad solo estafan a los acaudalados pacientes hasta sacarles la última moneda. Con la ayuda de su esposa tratará de volver a encontrar el verdadero propósito que tiene la medicina (Fotos 4 y 5 ).

\section{La educación como solución}

La trama que nos transmite la película nos lleva a reflexionar acerca del ejercicio de la medicina por parte de los profesionales en salud. Por lo expuesto, existen componentes que hacen que el médico en su labor de servicio se desvirtúe y en alguna forma puedan perder el propósito del significado de la medicina. $Y$ acerca de esos componentes, ¿Son inherentes a la situación? o

Rev. Med. Cine. 2021; 17 (1), 17-24 Ediciones Universidad de Salamanca / ॠ@@ J. Med. Mov., 2021; 17 (1), 17-24 
¿Son propias del médico que las practica (independientes a la situación)? o ¿ Quizá sean ambas? Sea como fuere, hay que enfatizar en soluciones para contrarrestar esto. Y como siempre ir hacia la base de todo, la educación médica.

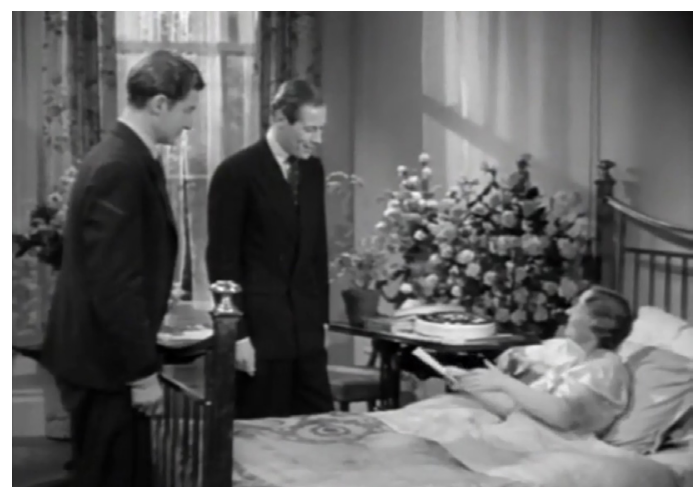

Foto 4. El Dr. Manson junto a un colega atiende a una paciente que no tiene ninguna sintomatología.

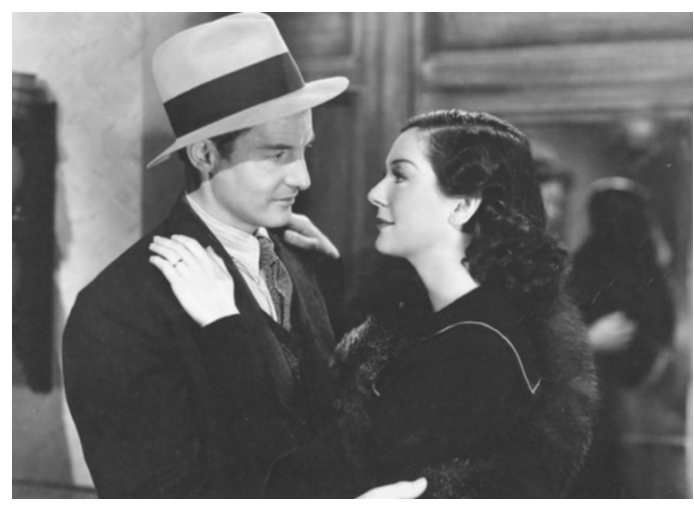

Foto 5. Christine dialoga con su esposo para que entienda nuevamente el propósito de la medicina.

La formación en medicina demanda una combinación especial de habilidades y comprensión. Y un pilar fundamental en este aprendizaje, aparte de los conocimientos técnicos y prácticos, son las competencias sobre la ética y humanidad. Dichos estudios promueven el desarrollo del discernimiento moral y juicio crítico frente a distintos escenarios en la atención y cuidado de los pacientes. En sí es la base de la relación médico-paciente pues solidifica la comunicación y confianza entre ambos agentes ${ }^{17,18}$. Asimismo, otro componente vital en la formación y vida del médico es la empatía, la cual lamentablemente la tendencia actual sugiere que este atributo se está perdiendo. Por consiguiente, se debe promover y potenciar los cursos de humanidades en las mallas curriculares de todas las carreras en ciencias de la salud, entre aquellas asignaturas destacan: Bioética, Historia de la medicina, Arte y medicina, Antropología, Filosofía, entre otros.

\section{Conclusión}

Frecuentemente pensamos que lo extraordinario es algo imposible de lograr, pero a veces la constancia de lo que consideramos "ordinario» es lo que lo vuelve extraordinario y visto de este modo, no es imposible de lograr. Simplemente cumplir bien nuestras funciones con perseverancia conlleva a una vida de excelencia, digna de ser un modelo a seguir. La experiencia, a veces, nos lleva a plasmar lo aprendido para exponerlo ante la sociedad, caso de esta película basada en la novela homónima de A.J. Cronin, cuya profesión antes de ser novelista fue la de doctor, y precisamente inició sus prácticas en una zona minera. Es probable que el sentido de la película haya sido llevar a una reflexión respecto a algunos elementos negativos que se dan durante el ejercicio de la medicina; hechos que pueden desarrollarse en cualquier medio, desde los entornos más oscuros de la condición de la humanidad exponiendo la casi imposible supervivencia de la moral. Se denuncia aquella mala praxis a través de la vida profesional del protagonista. Como sucedió con el Dr. Manson, nos exhibe la ligera línea que divide el buen ejercicio de la profesionalidad; es tan difícil llegar hasta la cúspide y tan fácil caer; pero es uno mismo quien siempre establece el final y siempre se puede resurgir o retomar el camino esté donde se esté. Hacia el final de la película se logra discernir el camino hacia la humildad y la honradez, siendo estos los mejores antídotos contra la soberbia; y 


\section{LA CIUDADELA (1938) DE KING VIDOR: LUCES Y SOMBRAS EN EL EJERCICIO DE LA PROFESIONALIDAD PALMER JOSÉ HERNÁNDEZ-YÉPEZ}

para la arrogancia, el mejor antídoto es la duda, por ello es importante en el ejercicio de la medicina el manejo de la incertidumbre. Todos estos valores deben cultivarse lo más tempranamente posible, incentivarlos desde el hogar, también en la formación primaria y secundaria; y afianzarlo durante la formación universitaria, en todas las carreras; así estaremos sentando las bases para una cultura de paz y tolerancia no solo en el ámbito profesional sino en cada uno de los aspectos de la vida. Quisiera terminar el presente artículo con las líneas finales de la película:

"Los médicos tienen una responsabilidad con la humanidad. No olvidemos las palabras del juramento hipocrático: En cualquier casa en la que yo entre lo haré siempre en beneficio del enfermo y huiré de todo error premeditado y de toda corrupción».

\section{Referencias}

1. Siurana-Aparisi JC. Los principios de la bioética y el surgimiento de una bioética intercultural. Veritas. 2010; (22): 121-57.

2. Saks M. Defining a profession: The role of knowledge and expertise. Professions and Professionalism. 2012; 2(1): 1-10.

3. Nuñez-Cortés JM. Valores del médico para un ejercicio de calidad: el profesionalismo. FEM. 2014; 17(suppl 1): S23-5.

4. Calman K. The profession of medicine. BMJ. 1994; 309(6962): 1140-43.

5. Yadavendu VK, Kumar D. Bioethics, medicine and society - a philosophical inquiry. Curr Sci. 2009; 97(8): 1128-36.

6 . Farrar J. Science, medicine, and society: A view from the Wellcome Trust. JAMA. 2015; 313(23): 2315-6.
7. Lee PR. The future of social medicine. J Urban Health. 1999; 76(2): 229-236.

8. Mckeown T, Lowe CR. An Introduction to Social Medicine. 1st ed. Oxford: Blackwell Scientific Publications; 1966.

9. Málaga-Rodríguez G, Sánchez-Mejía A. Medicina basada en la evidencia: Aportes a la práctica médica actual y dificultades para su implementación. Rev Med Hered. 2009; 20(2): 103-9.

10. Diderichsen F. Turning public health research into practice. Scand J Public Health. 2018; 46(22):3-4.

11. Raghav P. Strengthening occupational medicine to address challenges of occupational diseases: A priority. Indian J Public Health. 2017; 61(3): 153-4.

12. Smith DR, Guidotti TL. History of the Journal of Occupational and Environmental Medicine. J Occup Environ Med. 2016; 58(1): 1-2.

13. Berger AS. Arrogance among physicians. Acad Med. 2002; 77(2):145-7.

14. Ingelfinger FJ. Arrogance. N Engl J Med. 1980; 303(26): 1507-11.

15. Muula AS. Medicine and money: Friends or foe? Mens Sana Monogr. 2006; 4(1): 78-88.

16. Keshavjee S. Medicine and money: the ethical transformation of medical practice. Med Educ. 2004; 38(3): 271-5.

17. Doukas DJ, McCullough LB, Wear S. Perspective: Medical education in medical ethics and humanities as the foundation for developing medical professionalism. Acad Med. 2012; 87(3): 334-41.

18. Mendoza A. La relación médico paciente: consideraciones bioéticas. Rev Peru Ginecol Obstet. 2017; 63(4): 555-64.

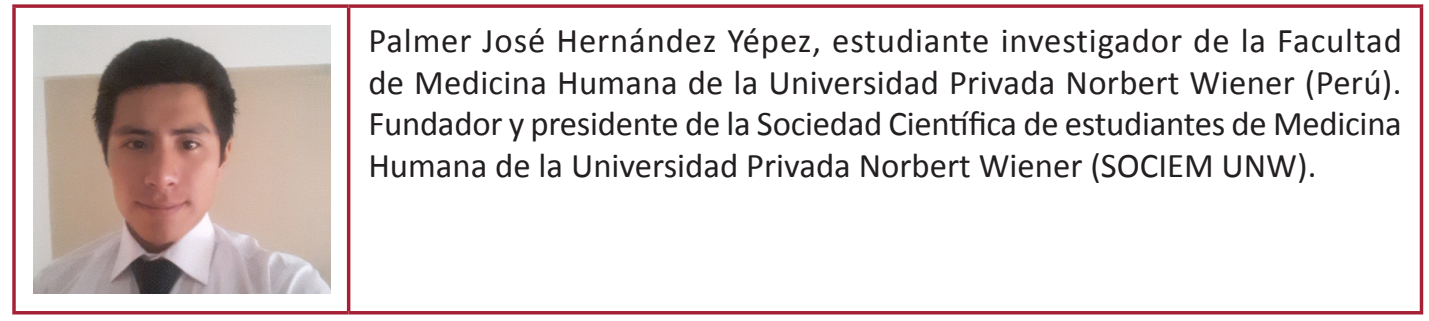

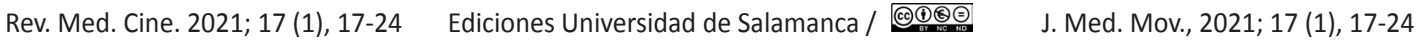

